\title{
A Delayed Presentation of Acute Generalized Exanthematous Pustulosis (AGEP)
}

\author{
Case Report
}

Laura Maurer BS ${ }^{1}$, Gonzalez-Estrada $\mathrm{A}^{2 *}$, Fernandez $\mathrm{J}^{2}$

${ }^{1}$ School of Medicine, Case Western Reserve University, Cleveland, OH, USA.

${ }^{2}$ Department of Allergy and Clinical Immunology, Cleveland Clinic, Cleveland OH, USA.

\begin{abstract}
Acute Generalized Exanthematous Pustulosis (AGEP) is a Type IVd hypersensitivity reaction that usually occurs within 1-5 days of drug exposure. The diagnosis is clinical and confirmed by skin biopsy. A scoring algorithm has been developed which assigns points to characteristic clinical signs in order to aid in the diagnosis. AGEP is a self-limited disease with a favorable prognosis in most cases. We present an unusual delayed presentation of AGEP secondary to hydroxycholoroquine.
\end{abstract}

Keywords: AGEP; Type IV Hypersensitivity; Adverse Drug Reaction; Hypersensitivity Delayed;

\section{*Corresponding Author:}

Alexei Gonzalez-Estrada MD,

Department of Allergy and Clinical Immunology, Cleveland Clinic Foundation, Cleveland OH, 9500 Euclid Ave, A90, Cleveland, OH, 44195, USA.

Tel: 2164446933

Fax: 2164452104

E-Mail: gonzala5@ccf.org

Recieved: March 25, 2015

Accepted: May 19, 2015

Published: May 22, 2015

Citation: Laura Maurer BS, Gonzalez-Estrada A, Fernandez J (2015) A Delayed Presentation of Acute Generalized Exanthematous Pustulosis (AGEP). Int J Clin Med Allergy. 03(1), 11-13. doi: http://dx.doi. org/10.19070/2332-2799-150004

Copyright: Gonzalez-Estrada $\mathbf{A}^{\odot}$ 2015. This is an open-access article distributed under the terms of the Creative Commons Attribution License, which permits unrestricted use, distribution and reproduction in any medium, provided the original author and source are credited.

\section{Introduction}

Acute Generalized Exanthematous Pustulosis (AGEP) occurs in 1-5 million cases per year, and has $2-5 \%$ mortality $[1,2]$. It is most frequently characterized by an acute episode of erythematous and edematous eruptions of sterile pustules within 1-5 days of drug exposure. AGEP has a female predominance [3] as well as a genetic susceptibility related to HLA B51, DR11, and DQ3 [4].

\section{Case Report}

A 70-year-old female with polymyalgia rheumatica on corticosteroids for 2 years presented with pruritic rash. The rash began on her face and spread to her scalp, chest, upper and lower extremities. The patient self- increased her prednisone from $7 \mathrm{mg}$ to $40 \mathrm{mg}$ a day with no relief and experienced subjective fevers,chills, malaise, increased thirst and anorexia requiring hospitalization. Medications on admission included loraze pam, hydrochlorothiazade/triamterene, fluticasone, tiotropium bromide, alendronate, ibuprofen, prednisone, and hydroxychloroquine. The patient was seen by Dermatology who noted diffuse erythematous pustular lesions (Figure I) on her scalp (Figure II), chest (Figure III), abdomen (Figure IV), and back (Figure V) and performed two skin biopsies. The differential diagnosis included Steven-Johnson's syndrome (SJS), drug rash with eosinophilia and systemic symptoms (DRESS), acute generalized exanthematous pustulosis (AGEP), pustular psoriasis, and infectious etiologies.

Laboratory workup was relevant for aleukocytosis with left shift (22,630 polymorphonuclear cells)and elevated CRP. Early signs of SJS and DRESS were monitored closely. Her biopsy results described pustular and erythematous lesions, spongiform subcorneal pustules with dermal acute and chronic inflammation with the presence of neutrophils. The cultures showed no growth. The skin biopsy along with the lack of eosinophils on blood smear favored a diagnosis of AGEP. Upon further questioning, she had started hydroxychloroquine three weeks prior to her cutaneous manifestations. Immediate skin prick testing was not performed. Patch testing may be considered in selected patients presenting with delayed type IV. However, this has not been validated and is not utilized at our Institution. Clinical history and histopathology supported an AGEP diagnosis despite an uncharacteristic threeweek span between inciting drug administration and reaction. Consequently, hydroxychloroquine was discontinued. Recovery occurred slowly over a three-week period and was complicated by S.auerus bacteremia secondary to skin integrity loss, requiring nafcillin treatment.

\section{Discussion}

AGEP is considered a Type IVd hypersensitivity reaction, characterized by drug specific T-cells secreting neutrophil attractant IL-8/CXCL8 $[5,6]$. Common causes of medication induced 
Figure 1. Diffuse erythematous pustular lesions.

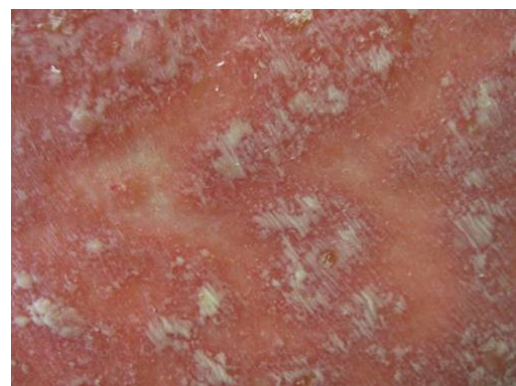

Figure 2. Scalp with erythematous pustular lesions.

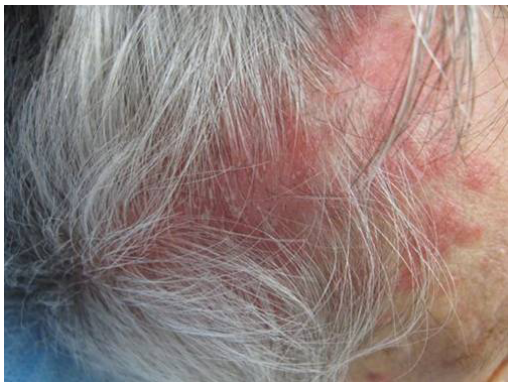

Figure 3. Chest with erythematous pustular lesions.

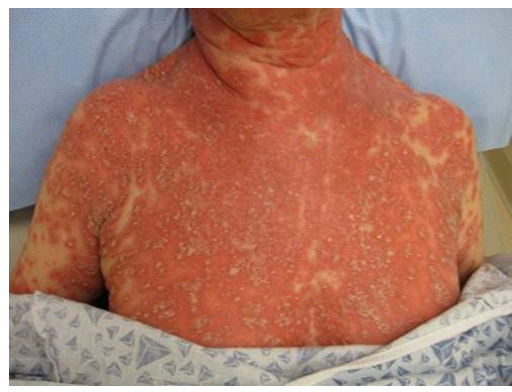

Figure 4. Abdomen with erythematous pustular lesions.

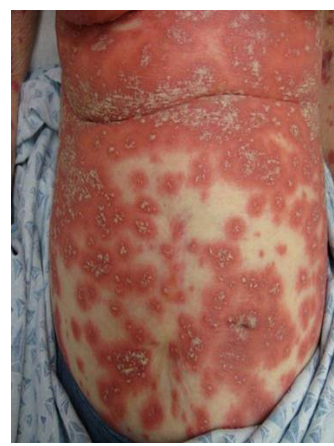

Figure 5. Upper back with erythematous pustular lesions.

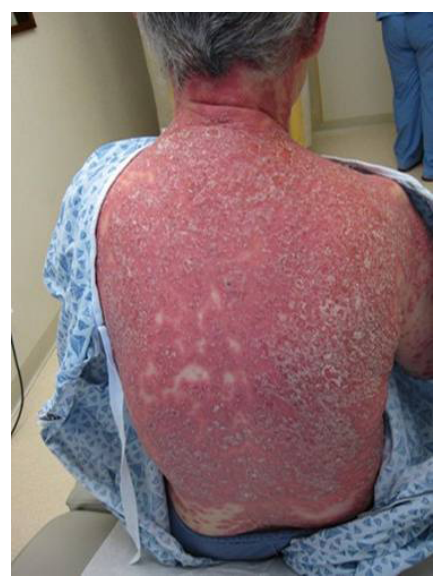


Table I. Medications that induce AGEP.

\begin{tabular}{|c|}
\hline Aminopenicillins \\
\hline Quinolones \\
\hline Anti-infective sulfonamides \\
\hline Hydroxychlorquine \\
\hline Pristinamycin \\
\hline Terbinafine \\
\hline Diltiazem \\
\hline Costicosteroids \\
\hline Macrolides \\
\hline NSAIDs \\
\hline Antiepileptics \\
\hline
\end{tabular}

AGEP [7] are shown in Table I. With regards to hydroxycholoroquine, the average time between drug initiation and the onset of reaction has been reported to be 12 days, with $78 \%$ of cases presenting in the first 15 days [3].

The diagnosis is dependent upon histopathology identifying subcorneal, intraepidermal pustules, edema of the papillary dermis, perivascular infiltrates with neutrophils and eosinophils, focal necrotic keratinocytes, and vasculitis [8]. This is frequently confused with generalized pustular psoriasis, with the key difference being the lack of acanthosis and papillomatosis in AGEP [7, 8]. A scoring algorithm has been developed which assigns points to characteristic clinical signs in order to aid in the diagnosis [1]. According to the algorithm, our patient had a highly probable diagnosis, losing points for delayed onset and slow recovery [1]. AGEP is a self-limited disease with a favorable prognosis in most cases. The recommended treatment consists of withdrawal of the suspected drug and supportive therapy [1]. Secondary skin infections are a common complication in the elderly and immune compromised patients.

\section{References}

[1]. Sidoroff A, Halevy S, Bavinck JN, Bavinck JN, Vaillant L, Roujeau JC (2001) Acute generalized exanthematous pustulosis (AGEP)--a clinical reaction pattern.J Cutan Pathol. 28(3):113.

[2]. Saissi EH, Beau-Salinas F, Jonville-Béra AP, Lorette G, Autret-Leca E (2003) Centres Regionaux de Pharmacovigilance. Drugs associated with acute generalized exanthematic pustulosis. Ann Dermatol Venereol. 2003 Jun;130 (6-7):612-8.

[3]. Sidoroff A, Dunant A, Viboud C, Halevy S, Bavinck JN, Naldi L, et al (2007). Risk factors for acute generalized exanthematous pustulosis (AGEP)results of a multinational case-control study (EuroSCAR).Br J Dermatol. 157(5):989.
[4]. Bernard P, (1995) Lizeaux-Parneix V, Miossec V. HLA et pre-dis $\neg$ position genetique dans les pustuloses exanthematiques (PEAG) et les exanthemes maculo-papuleux (EMP). Ann Dermatol Venerol;122:S38.

[5]. Britschgi M, Pichler WJ (2002). Acute generalized exanthematous pustulosis, a clue to neutrophil-mediated inflammatory processes orchestrated by $\mathrm{T}$ cells. Curr Opin Allergy Clin Immunol. Aug 2(4):325-31.

[6]. Britschgi M, Steiner UC, Schmid S, Depta JP, Senti G, Bircher A, et al (2001). T-cell involvement in drug-induced acute generalized exanthematous pustulosis. J Clin Invest.;107(11):1433.

[7]. Sidoroff A, Dunant A, Viboud C, Halevy S, Bavinck JN, Naldi L, et al (2007): Risk factors for acute generalized exanthematous pustulosis (AGEP)-results of a multinational case-control study (EuroSCA).Br J Dermatol. Nov 157(5):898-96.

[8]. Anderson E, Daft K, Lee R, Elenitsas R (2006) Update in dermatopathology. Am J Clin Pathol.125 Suppl 1: S50-S70.

[9]. Roujeau J, Bioulac-Sage P, Bourseau C, Guillaume JC, Bernard P, Lok C, et al (1991) Acute Generalized Exanthematous Pustulosis: Analysis of 63 Cases. Arch Dermatol.127(9):1333-1338.

[10]. Fernando SL (2012) Acute generalised exanthematous pustulosis. Australia J Dermatol. 53(2):87-92. 